\title{
RESEARCH
}

\section{miR-23a-3p increases endometrial receptivity via CUL3 during embryo implantation}

\author{
Kai Huang', Gezi Chen², Wenqian Fan' ${ }^{1}$ and Linli Hu \\ ${ }^{1}$ Center for Reproductive Medicine, Henan Key Laboratory of Reproduction and Genetics, Henan Provincial Obstetrical and Gynecological Diseases \\ (Reproductive Medicine) Clinical Research Center, Henan Engineering Laboratory of Preimplantation Genetic Diagnosis and Screening, The First Affiliated \\ Hospital of Zhengzhou University, Zhengzhou, China \\ 2Department of Obstetrics, The First Affiliated Hospital of Zhengzhou University, Zhengzhou, China
}

Correspondence should be addressed to L Hu: acceptzzu@126.com

\begin{abstract}
A receptive endometrium is required in a successful embryo implantation. The ubiquitination-induced $\beta$-catenin degradation is related to the implantation failure. This study aimed to elucidate whether miR-23a-3p regulates endometrial receptivity via the modulation of $\beta$-catenin ubiquitination.The expressions of miR-23a-3p and CUL3 were detected in endometrial epithelial cells (EECs) isolated from pregnant mice and in hormone-induced EEC-like Ishikawa cells. The ubiquitination experiment was performed to explore the effect of CUL3 and miR-23a-3p on $\beta$-catenin ubiquitination level. The trophoblast attachment was detected by co-culturing JAR (choriocarcinoma cell line) spheroids with Ishikawa cell monolayers. miR-23a-3p was upregulated while CUL3 was downregulated in EECs at day 4 after pregnancy compared with day 1 , as well as in hormone-induced Ishikawa cells. miR-23a-3p positively regulated the protein level of $\beta$-catenin without affecting the mRNA level. The ubiquitination and degradation of $\beta$-catenin was suppressed by miR-23a-3p, while it was promoted by CUL3. Immunoprecipitation confirmed the binding between CUL3 and $\beta$-catenin. Luciferase reporter assay confirmed the target relationship between miR-23a-3p and CUL3. The ubiquitination of $\beta$-catenin was modulated by the miR-23a-3p/CUL3 pathway. The overexpression of miR-23a-3p promoted JAR spheroid attachments in Ishikawa cells. miR-23a-3p is beneficial for the endometrial receptivity and embryo implantation, whose mechanism is partly through the modulation of CUL3/ $\beta$-catenin.
\end{abstract}

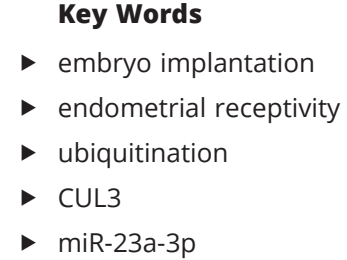

Journal of Molecular Endocrinology (2020) 65, 35-44

\section{Introduction}

Embryo implantation is the first step to establish a successful pregnancy, which requires a normal endometrial receptivity and a functional embryo (Garrido-Gomez et al. 2012). With the attachment between trophoblasts in embryo and endometrial epithelial cells (EECs) in the uterus, a synchronized dialogue is started between embryonic and maternal tissues (Cagil et al. 2011). During this process, estrogen (E) and progesterone (P) secreted by the ovaries play an important role, which promote the transformation of endometrium toward secretory and receptive condition in order to receive the embryo (Lim \& Wang 2010). At the same time, many molecules, including leukemia inhibitory factor (LIF), integrin $\alpha v \beta 3$, and osteopontin (OPN), were released by EECs to enhance the endometrial receptivity (Schmitz et al. 2014, Zheng et al. 2016, Berneau et al. 2019). Therefore, the 
EEC-mediated endometrial receptivity plays a critical role in embryo implantation, whose mechanisms have been largely unknown.

The Wnt- $\beta$-catenin signaling pathway is well identified in regulating embryo implantation process (Sonderegger et al. 2010). As reported, the Wnt- $\beta$-catenin signaling pathway is critically involved in trophoblast implantation and adhesion to maternal uterine tissues (Hess et al. 2007, Liu et al. 2010). Both Wnt and $\beta$-catenin proteins are key components of the Wnt- $\beta$-catenin signaling pathway. When the Wnt ligand associates with the receptors on the cell surface, the Wnt- $\beta$-catenin signaling pathway is turned on and large amounts of $\beta$-catenin protein transport into nucleus, thus exerting the transcriptional functions. When the Wnt ligand is blocked, the Wnt$\beta$-catenin signaling pathway is turned off and $\beta$-catenin proteins are ubiquitinated, thus gradually being degraded by the proteasomes (Liu et al. 2002). Therefore, the inhibition of $\beta$-catenin ubiquitination is beneficial for the maintenance of endometrial receptivity and the promotion of trophoblast implantation. CUL3, also known as Cullin 3, modulates the process of ubiquitination and is reported to negatively regulate $\beta$-catenin protein expression via increasing its ubiquitination (Angers et al. 2006). However, whether CUL3 is involved in regulating EEC-mediated endometrial receptivity by interacting with $\beta$-catenin remains unclear.

miRNAs are a group of endogenous ncRNAs encoded by the genome of almost all species (Wang et al. 2018). The mature miRNA is about 19-25 nucleotides in length and is derived from the initial transcript pri-miRNA in the nucleus. By binding with the 3'UTR of targeted mRNA, miRNAs exert the regulatory function via degrading the target mRNAs (Beermann et al. 2016). Several miRNAs have been identified to modulate the embryo implantation process, such as miR-199a, miR-101, and miR-21 (Chakrabarty et al. 2007, $\mathrm{Hu}$ et al. 2008). miR-23a-3p is reported to regulate cell proliferation and differentiation in various types of cells (Godfrey et al. 2018, Zhao et al. 2019). Although it is rarely reported to regulate the embryo implantation process, a study conducted by Yang et al. (2018) suggested that miR-23a-3p was lowly expressed in recurrent miscarriage patients and that lower serum level of miR-23a-3p was related to the failure of embryo implantation. Notably, we found that there are predicted binding sites between miR-23a-3p and CUL3 mRNA, suggesting the potential effect of miR-23a-3p on CUL3 expression.

In the current study, the expressions of miR-23a-3p and CUL3 were detected in EECs isolated from pregnant mice and in hormone-induced EEC-like Ishikawa cells. Meanwhile, the effect of miR-23a-3p/CUL3 on ubiquitination of $\beta$-catenin was also elucidated, hoping to provide the evidence for the mechanisms underlying EEC-mediated endometrial receptivity during embryo implantation.

\section{Materials and methods}

\section{Isolation of EECs from pregnant mice}

The female Kunming mice (6-week old) were bought from the Shanghai Lab Animal Research Center (Shanghai, China). They were divided into two groups according to the pregnant date, the 1-day group (1 day after the pregnancy) and the 4-day group (4 days after the pregnancy). There were six mice in each group. EECs were isolated from uterine tissues from the two groups as previously described (Pan et al. 2017). Briefly, the uterine horns were cut into small pieces on ice, followed by washing twice using Hanks' balanced salt solution (HBSS) which was deprived with $\mathrm{Mg}^{2+} / \mathrm{Ca}^{2+}$. To digest the tissues, $7 \mathrm{~g} / \mathrm{L}$ trypsin and $18 \mathrm{~g} / \mathrm{L}$ dispase (Sigma) were added for $30 \mathrm{~min}$ at $4{ }^{\circ} \mathrm{C}$ before being placed for another $30 \mathrm{~min}$ at room temperature. After collecting the epithelial plaques, we washed the collected cells twice using HBSS. Then they were cultured in 12-well plates with complete media (10\% charcoal-stripped fetal bovine serum (FBS), 1\% penicillin/ streptomycin, and phenol-red-free DMEM/F12).

\section{Culture and treatment of Ishikawa cells}

The immortalized human uterine endometrial adenocarcinoma cell line (Ishikawa) was bought from the ATCC. The culture medium consisted of RPMI 1640 supplemented with $5 \% \mathrm{FBS}$. Cells were stored in a standard tissue culture incubator at $37{ }^{\circ} \mathrm{C}$, with $5 \% \mathrm{CO}_{2}$ and $95 \%$ humidity. The medium was changed to phenol-red-free RPMI 1640 containing 5\% charcoal-stripped FBS $24 \mathrm{~h}$ prior to cell treatment. Ishikawa cells were treated with different concentrations of $17 \beta$-estradiol $\left(\mathrm{E} ; 10^{-9}, 10^{-8}\right.$, and $10^{-7} \mathrm{~mol} / \mathrm{L}$ ), different concentrations of progesterone (P; $10^{-6}, 10^{-5}$, and $10^{-4} \mathrm{~mol} / \mathrm{L}$ ), or combinedly treated with $10^{-8} \mathrm{~mol} / \mathrm{L} \mathrm{E}+10^{-6} \mathrm{~mol} / \mathrm{L} \mathrm{P}$ (Zhu et al. 2013). Ishikawa cells were treated with these hormones for $96 \mathrm{~h}$.

\section{Quantitative real-time PCR (qRT-PCR)}

To extract RNAs from cells and tissues, the TRIzol reagent (Thermo Fisher Scientific) was used. To obtain cDNAs 
reversely transcribed from RNA, the RT Kit (Thermo Fisher Scientific) was used. The SYBR-Green MicroRNA Kit and SYBR Green PCR Kit (Takara) were used to perform the qRT-PCR assay, which was conducted on ABI 7500 FAST Real-Time PCR System (Thermo Fisher Scientific). The expression levels were determined using $2^{-\Delta \Delta C t}$ method. U6 was used as the control for miR-23a-3p, and GAPDH was used as the control for CUL3, Wnt1, and CTNNB1 mRNA.

\section{Western blot analysis}

Proteins were extracted by the AllPrep Protein/RNA/DNA Mini Kit (Qiagen). The primary antibodies used in this study included anti-CUL3 (ab75851, 1/20,000, Abcam), anti-Wnt1 (ab15251, 1/5000, Abcam), anti- $\beta$-catenin (ab6302, 1/4000, Abcam), anti-LIF (ab113262, $1 \mu \mathrm{g} / \mathrm{mL}$, Abcam), anti-Integrin $\alpha \mathrm{v}$ (ab179475, 1/5000, Abcam), anti-integrin $\beta 3$ (ab119992, 1/1000, Abcam), anti-OPN (ab75285, 1/500, Abcam), and ani- $\beta$-actin (ab8226, $1 / 500$, Abcam). The secondary antibodies used included horseradish peroxidase-conjugated anti-rabbit IgG (ab205718, 1/2000, Abcam). Expression was visualized with the ChemiDoc XRS system (Bio-Rad) and the ECL enhanced reagent (Thermo Fisher Scientific).

\section{Ishikawa cell transfection}

The miR-23a-3p mimic/inhibitor and their negative controls (pre-NC and NC) were purchased from Ribobio Technology (Guangzhou, Guangdong, China). The CUL3 overexpression plasmid vector (pcDNA-CUL3) and its negative control (pcDNA), as well as the siRNA against CUL3 (siRNA-CUL3) and its negative control (siRNA) were constructed by Genechem Corporation (Shanghai, China). Lipofectamine 2000 (Thermo Fisher Scientific) was used in cell transfection.

\section{Ubiquitination assay and cycloheximide (CHX) experiment}

The effect of miR-23a-3p/CUL3 on the ubiquitination of $\beta$-catenin was analyzed by co-transfecting Ishikawa cells with HA-Ub and miR-23a-3p mimic/pcDNA-CUL3, with MG132 $(10 \mu \mathrm{g} / \mathrm{mL})$ treatment for $2 \mathrm{~h}$ before detection. The cell lysates were immunoprecipitated with anti- $\beta$ catenin, and the ubiquitination levels of $\beta$-catenin were detected by immunoblotting with anti-HA antibody.

For the CHX experiment, Ishikawa cells were transfected with miR-23a-3p mimic/pcDNA-CUL3, followed by the addition of $50 \mu \mathrm{g} / \mathrm{mL} \mathrm{CHX}$. The relative expression of $\beta$-catenin at $0,6,9,12$, and $24 \mathrm{~h}$ after CHX treatment was detected by using Western blot analysis.

\section{Immunoprecipitation assay}

The immunoprecipitation assay is conducted by microMACS protein A/G microbeads magnetic separation (MedChemExpress, Monmouth Junction, NJ, USA). In brief, total protein $(100 \mu \mathrm{g})$ was incubated on ice for 30 min with protein $\mathrm{A} / \mathrm{G}$ microbeads $(20 \mu \mathrm{L})$ and $2 \mu \mathrm{g}$ of the IgG or anti- $\beta$-catenin. During a magnetic field, the magnetically labeled immune complex was passed over a microcolumn, followed by washing with the lysis buffer. Then the sodium dodecyl sulfate gel loading buffer was used to elute the immunoprecipitated proteins from the column, followed by immunoblotting with $\beta$-catenin antibody or CUL3 antibody.

\section{Luciferase reporter assay}

CUL3 3'UTR which contains the predicted binding sites of miR-23a-3p was amplified by using PCR. Mutant CUL3 3 'UTR was synthesized by Invitrogen. The products were cloned into the psi-CHECK2 vector (Promega). 293T cells were plated in 96-well plates, followed by the transfection with 100 ng mutant (Mut) or WT CUL3 3'UTR vectors, together with 30 pmol miR-23a mimic/inhibitor. Cells were harvested for the luciferase assay after $48 \mathrm{~h}$. Then, we used Dual-Luciferase Reporter Assay System (Promega) to evaluate the Renilla and firefly luciferases activities, and the value was normalized to the firefly luciferase activity.

\section{JAR spheroid attachment to Ishikawa cell monolayers}

The human choriocarcinoma cell line (JAR) was purchased from ATCC and was cultured in RPMI 1640 medium (5\% FBS) in a standard tissue culture incubator. The co-culture of JAR spheroids and Ishikawa cells was performed as previously described (Li et al. 2017). The JAR cells were then trypsinized, washed, and seeded in a 6-well plate at a concentration of $2 \times 10^{5}$ cells per well. Then they were shaken overnight. Ishikawa cells were plated in 12-well plates $\left(2 \times 10^{5}\right.$ cells per well). Ishikawa cells were transfected with miR-23a-3p mimic followed by the treatment of $10^{-8} \mathrm{~mol} / \mathrm{L} 17 \beta$-estradiol (E) for 24 h. The JAR spheroids ( 45 spheroids/dish) were transferred onto the surface of Ishikawa cell monolayers for $7 \mathrm{~h}$ at $37{ }^{\circ} \mathrm{C}$ with $5 \% \mathrm{CO}_{2}$. Then the medium was removed 
and the plates were centrifuged for $10 \mathrm{~min}$ at $10 \mathrm{~g}$. The attached spheroids were counted. The attachment rate was calculated (attached spheroid number/seeded spheroid number).

\section{Immunofluorescence analysis}

The JAR cells were probed with the antibody against PCNA (1:100, $2 \mu \mathrm{g} / \mathrm{mL}$, ab92552, Abcam). Then they were incubated with IgG Alexa 488 (ab150077, Abcam) for $1 \mathrm{~h}$ at a dilution of 1:200. After being washed by $0.1 \%$ BSA in PBS, the cells were overlaid with DAPI. The images were taken at 7 and $24 \mathrm{~h}$ after the co-culture by using a confocal microscope (Carl Zeiss). The spheroid expansion was calculated as the ratio of the spheroid diameter at $24 \mathrm{~h}$ to the spheroid diameter at $7 \mathrm{~h}$.

\section{Statistical analysis}

Data are shown as mean \pm s.E.M. To compare the differences between the two groups, the Student's $t$-test was used. To compare the differences among multiple groups, the one-way ANOVA and Tukey's post hoc test was used, and $P<0.05$ was considered significant.

\section{Results}

miR-23a-3p is upregulated while CUL3 is downregulated in EECs during pregnancy and in E/P-induced Ishikawa cells

EECs were isolated from uterine tissues of pregnant mice at day 1 and day 4, then the expressions of miR-23a-3p and CUL3 were detected using qRT-PCR and Western blot analysis. As indicated in the 4-day group, the relative expression of miR-23a-3p was significantly upregulated compared with the non-pregnant group and the 1-day group (Fig. $1 \mathrm{~A}, P<0.01$ ), and the mRNA expression and the protein expression of CUL3 were downregulated (Fig. 1B, $P<0.05$ ). On the other hand, the expressions of miR-23a-3p and CUL3 were also detected in Ishikawa cells which were treated with different concentrations of estradiol (E) and/or progesterone (P). With the increasing concentration of $\mathrm{E}$ (from $10^{-9}$ to $10^{-7} \mathrm{~mol} / \mathrm{L}$ ), miR-23a-3p expression did not show a concentration-dependent manner, while with the increasing concentration of $\mathrm{P}$ (from $10^{-6}$ to $10^{-4} \mathrm{~mol} / \mathrm{L}$ ), miR-23a-3p showed a concentration-dependent upregulation in Ishikawa cells (Fig. 1C). In addition, the combined treatment of E $\left(10^{-8} \mathrm{~mol} / \mathrm{L}\right)+\mathrm{P} \quad\left(10^{-6} \mathrm{~mol} / \mathrm{L}\right)$ caused a marked
A

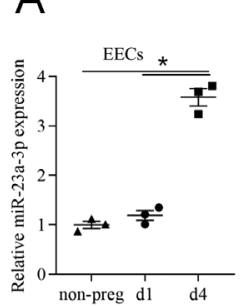

B

C
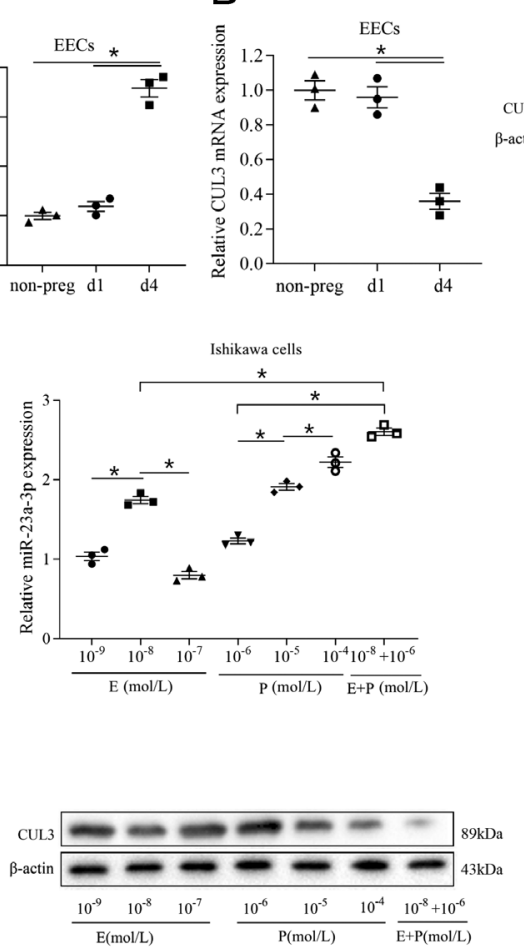
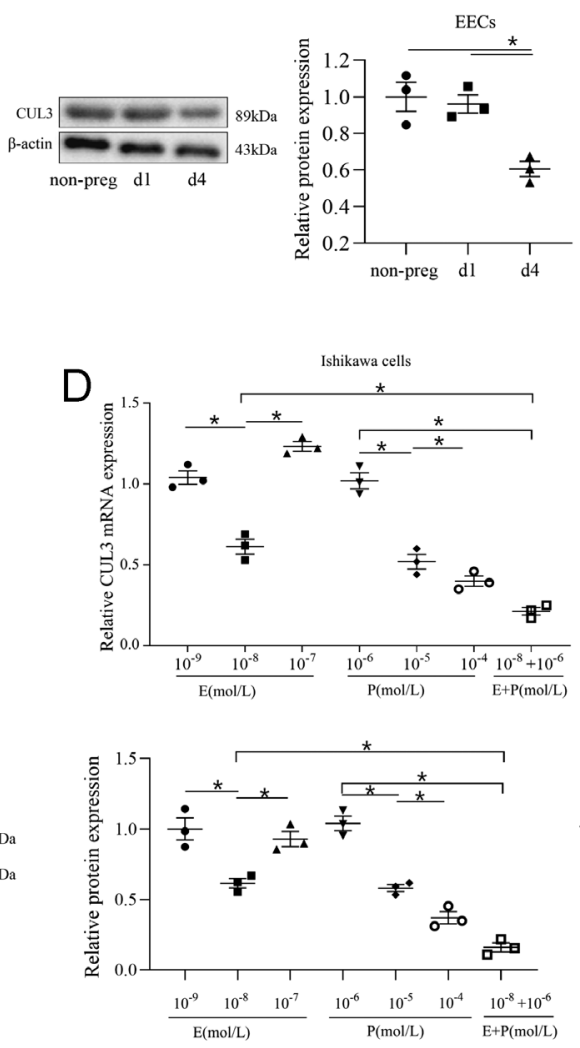

\section{Figure 1}

Expressions of miR-23a-3p and CUL3 in EECS during pregnancy and E/P-induced Ishikawa cells. EECS were isolated from uterine tissues of non-pregnant mice $(n=6)$ and pregnant mice at day $1(n=6)$ and day $4(n=6)$, then the expressions of miR-23a-3p (A) and the mRNA and protein expressions of CUL3 (B) were detected using qRT-PCR and Western blot analysis. Ishikawa cells were treated with different concentrations of estradiol (E) and/or progesterone $(P)$ for $96 \mathrm{~h}$. The expressions of miR-23a-3p (C) and the mRNA and protein expressions of CUL3 (D) were detected using qRT-PCR and Western blot analysis. ${ }^{*} P<0.05$. 
enhancement of miR-23a-3p in comparison with E $\left(10^{-8} \mathrm{~mol} / \mathrm{L}\right)$ or $\mathrm{P}\left(10^{-6} \mathrm{~mol} / \mathrm{L}\right)$ treated alone (Fig. $\left.1 \mathrm{C}\right)$. Similarly, with the increasing concentration of $\mathrm{P}$ (from $10^{-6}$ to $\left.10^{-4} \mathrm{~mol} / \mathrm{L}\right)$, CUL3 showed a concentrationdependent downregulation in Ishikawa cells, and the combined treatment of $\mathrm{E}\left(10^{-8} \mathrm{~mol} / \mathrm{L}\right)+\mathrm{P}\left(10^{-6} \mathrm{~mol} / \mathrm{L}\right)$ caused a significant reduction of CUL3 in comparison with E $\left(10^{-8} \mathrm{~mol} / \mathrm{L}\right)$ or $\mathrm{P}\left(10^{-6} \mathrm{~mol} / \mathrm{L}\right)$ treated alone (Fig. 1D).

\section{miR-23a-3p negatively regulates the expression of CUL3}

The online database Targetscan (www.targetscan.org) and RAID (http://www.rna-society.org/raid2/) predicted the binding between miR-23a-3p and CUL3 3'UTR in several species (Fig. 2A). After transfecting miR-23a-3p mimic/inhibitor into Ishikawa cells, we confirmed the transfection efficacy as shown in Fig. 2B. We mutated the potential binding sites and cloned them into a plasmid reporter vector. The results of the luciferase reporter assay indicated that overexpressing miR-23a-3p reduced the relative luciferase activity in the unmutated group (the WT CUL3 group), while knocking down miR-23a-3p increased the relative luciferase activity in the unmutated group (the WT CUL3 group). Both overexpressing or knocking down miR-23a-3p did not change the luciferase activity in the mutated group (the Mut CUL3 group) (Fig. 2C). Meanwhile, the simultaneous knockdown of miR-23a-3p and CUL3 reduced the enhancement of CUL3 mRNA and protein levels which were raised by miR-23a-3p knockdown (Fig. 2D). Ishikawa cells were transfected with miR-23a-3p mimic or co-transfected with CUL3 overexpressing plasmid (pcDNA-CUL3). The co-transfection restored the defect of both mRNA and protein expressions of CUL3 (Fig. 2E). These data indicated that miR-23a-3p negatively regulated the expression of CUL3 in Ishikawa cells.

\section{The role of miR-23a-3p on the Wnt- $\beta$-catenin signaling pathway}

The expressions of the key components of the Wnt- $\beta$ catenin signaling pathway, including Wnt1 and $\beta$-catenin, were detected after transfection. The result showed that both mRNA and protein levels of Wnt1 was not significantly changed after miR-23a-3p overexpression or knockdown (Fig. 3A). The mRNA level of CTNNB1 (the encoding gene of $\beta$-catenin) was not much changed, whereas the protein level of $\beta$-catenin was upregulated by miR-23a-3p overexpression and was downregulated

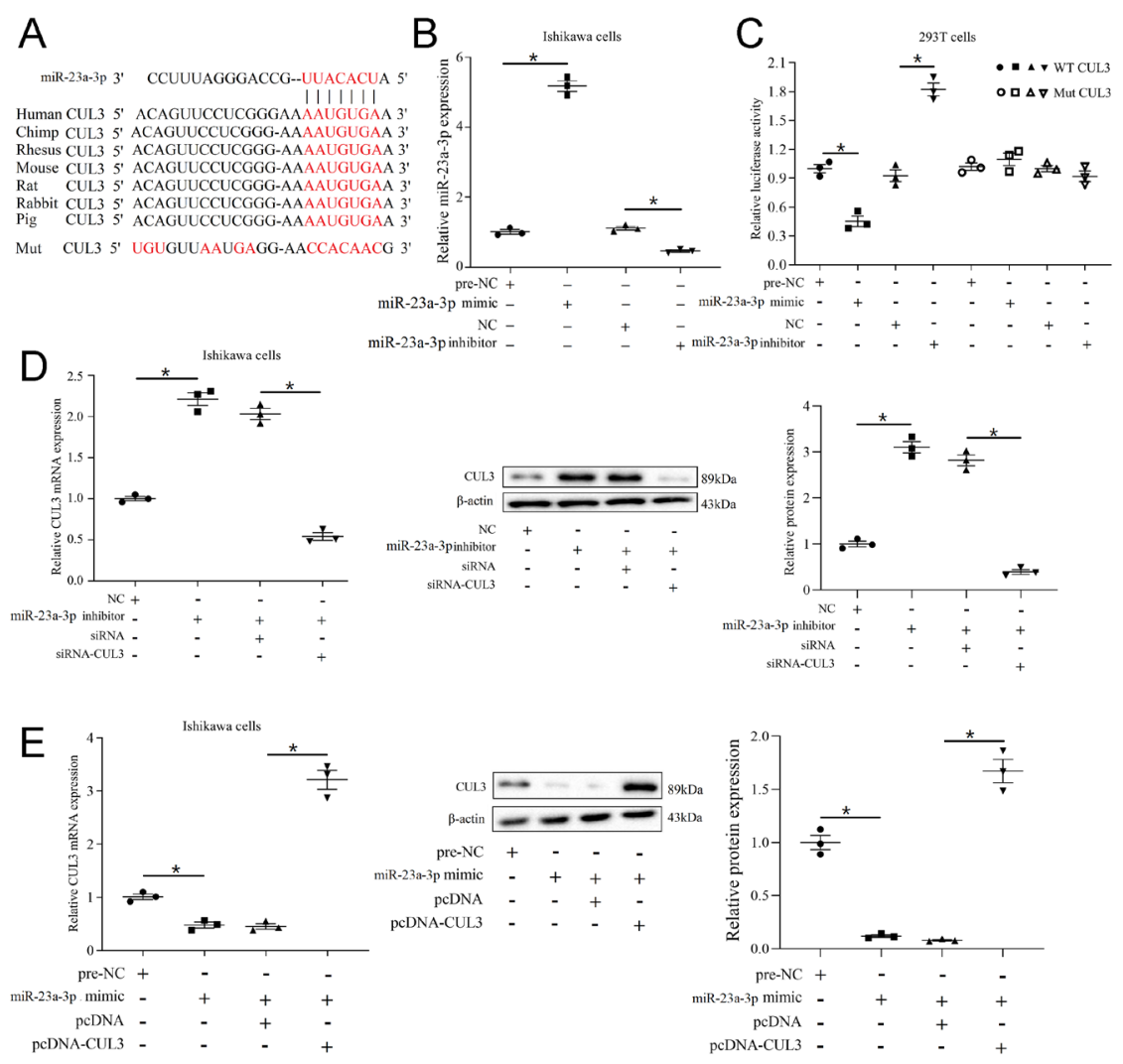

Figure 2

miR-23a-3p negatively regulates the expression of CUL3. (A) The predicted binding sites between miR-23a-3p and CUL3 3'UTR via online database Targetscan (www.targetscan.org) and RAID (http:// www.rna-society.org/raid2/). (B) Ishikawa cells were transfected with miR-23a-3p mimic/inhibitor for $48 \mathrm{~h}$ to overexpress/knockdown miR-23a-3p. The transfection efficacy was confirmed using qRT-PCR. (C) The 293T cells were transfected with miR-23a-3p mimic/inhibitor together with WT or mutant (Mut) CUL3 3'UTR reporter vector. The relative luciferase activity was detected using luciferase reporter assay. (D) Ishikawa cells were transfected with miR-23a-3p inhibitor or co-transfected with small interfering RNA (siRNA) against CUL3 (siRNA-CUL3). The MRNA and the protein levels of CUL3 were detected using qRT-PCR and Western blot analysis, respectively. (E) Ishikawa cells were transfected with miR-23a-3p mimic or co-transfected with CUL3 overexpressing plasmid (pcDNA-CUL3). The mRNA and the protein levels of CUL3 were detected using qRT-PCR and Western blot analysis, respectively. ${ }^{*} P<0.05$. 
A
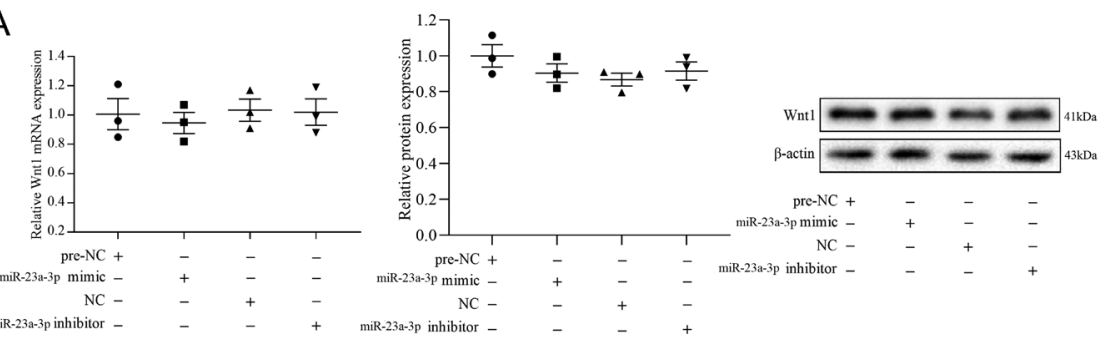

B
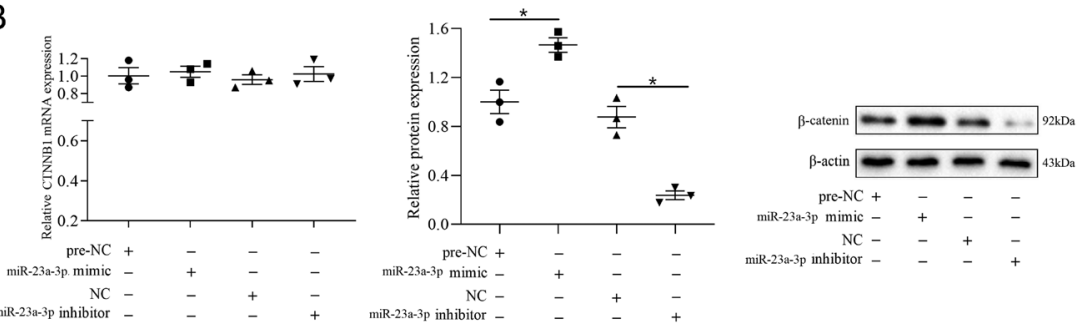

\section{Figure 3}

The role of miR-23a-3p on the Wnt- $\beta$-catenin signaling pathway. Ishikawa cells were transfected with miR-23a-3p mimic/inhibitor for $48 \mathrm{~h}$ to overexpress/knockdown miR-23a-3p. (A) The mRNA and protein expressions of Wnt1 were detected using qRT-PCR and Western blot analysis. (B) The mRNA level of CTNNB1 (the encoding gene of $\beta$-catenin) and the protein level of $\beta$-catenin were detected using qRT-PCR and Western blot analysis. ${ }^{*} P<0.05$.

by miR-23a-3p knockdown (Fig. 3B). These data indicated that miR-23a-3p affect the Wnt- $\beta$-catenin signaling pathway via positively regulating the protein expression of $\beta$-catenin in Ishikawa cells.

\section{miR-23a-3p suppresses ubiquitination of $\beta$-catenin via inhibiting CUL3 expression}

Given that the protein level of $\beta$-catenin was regulated by miR-23a-3p, we further investigated the effect of miR-23a-3p on the ubiquitination of $\beta$-catenin. The IP assay indicated the binding between $\beta$-catenin and CUL3 (Fig. 4A). After overexpressing miR-23a-3p in Ishikawa cells, the ubiquitin-modified $\beta$-catenin was reduced (Fig. 4B). Meanwhile, after inhibiting protein synthesis via $\mathrm{CHX}$, the degradation of $\beta$-catenin was suppressed by miR-23a-3p overexpression (Fig. 4C), suggesting miR-23a-3p inhibited the degradation of $\beta$-catenin via suppressing its ubiquitination. After overexpressing CUL3 in Ishikawa cells, the mRNA expression of CTNNB1 (the encoding gene of $\beta$-catenin) was not significantly changed, whereas the protein level of $\beta$-catenin was downregulated (Fig. 4D), indicating that CUL3 modulated the $\beta$-catenin protein expression. In addition, CUL3 overexpression promoted the $\beta$-catenin ubiquitination (Fig. 4E) and the $\beta$-catenin degradation (Fig. 4F). In addition, the simultaneous knockdown of miR-23a-3p and CUL3 negated the promotion of $\beta$-catenin ubiquitination caused by miR-23a-3p knockdown (Fig. $4 \mathrm{G})$. These findings demonstrated that miR-23a-3p suppressed ubiquitination of $\beta$-catenin via inhibiting CUL3 expression.
miR-23a-3p promotes JAR spheroid attachments in Ishikawa cells via suppressing CUL3 expression and increasing $\beta$-catenin expression

Ishikawa cells were transfected with miR-23a-3p mimic or co-transfected with CUL3 overexpressing plasmid (pcDNA-CUL3). The overexpression of miR-23a-3p increased the protein levels of $\beta$-catenin, LIF, integrin $\alpha \mathrm{v}$, integrin $\beta 3$, and OPN, while such response was negated by the co-transfection with pcDNA-CUL3 (Fig. 5A), suggesting endometrial receptivity was modulated by miR-23a-3p/CUL3 pathway. Furthermore, overexpressing miR-23a-3p markedly increased JAR spheroid attachments in Ishikawa cells (Fig. 5B) and promoted JAR spheroid expansion at $24 \mathrm{~h}$ in comparison with $7 \mathrm{~h}$ (Fig. 5C). Taken together, our findings indicated that miR-23a-3p promoted JAR spheroid attachments in Ishikawa cells via targeting CUL3 and suppressing the ubiquitination of $\beta$-catenin.

\section{Discussion}

A complex series of events are involved in the embryo implantation, causing the establishment of an initial connection between embryonic and maternal tissues (Diedrich et al. 2007). The failure of embryo implantation is often related to the poor quality of embryo and poor endometrial receptivity. As the endometrial receptivity is relatively easy to manually intervene than embryo quality, researchers have been focused on the mechanisms of endometrial receptivity in recent years (Haouzi et al. 2009). In the current study, we found a novel regulatory molecule, miR-23a-3p that modulates endometrial 

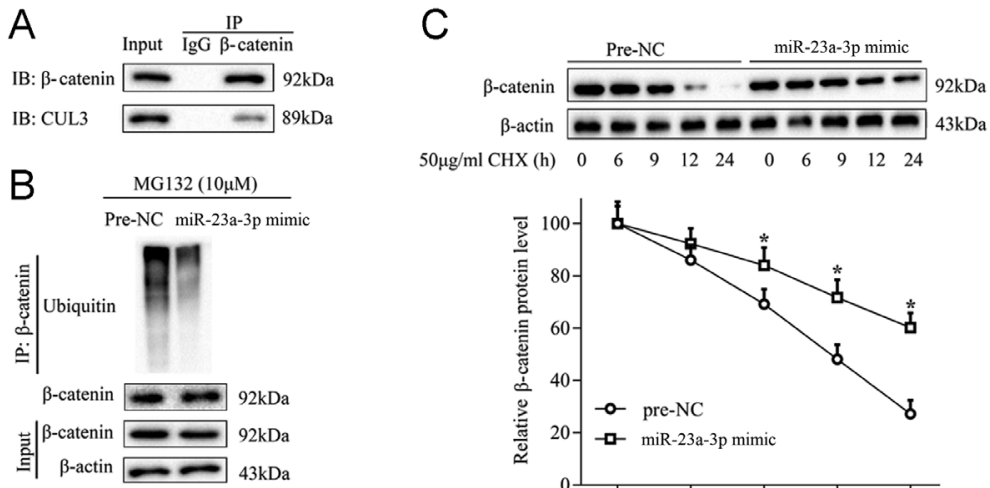

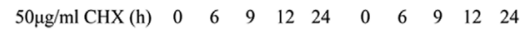
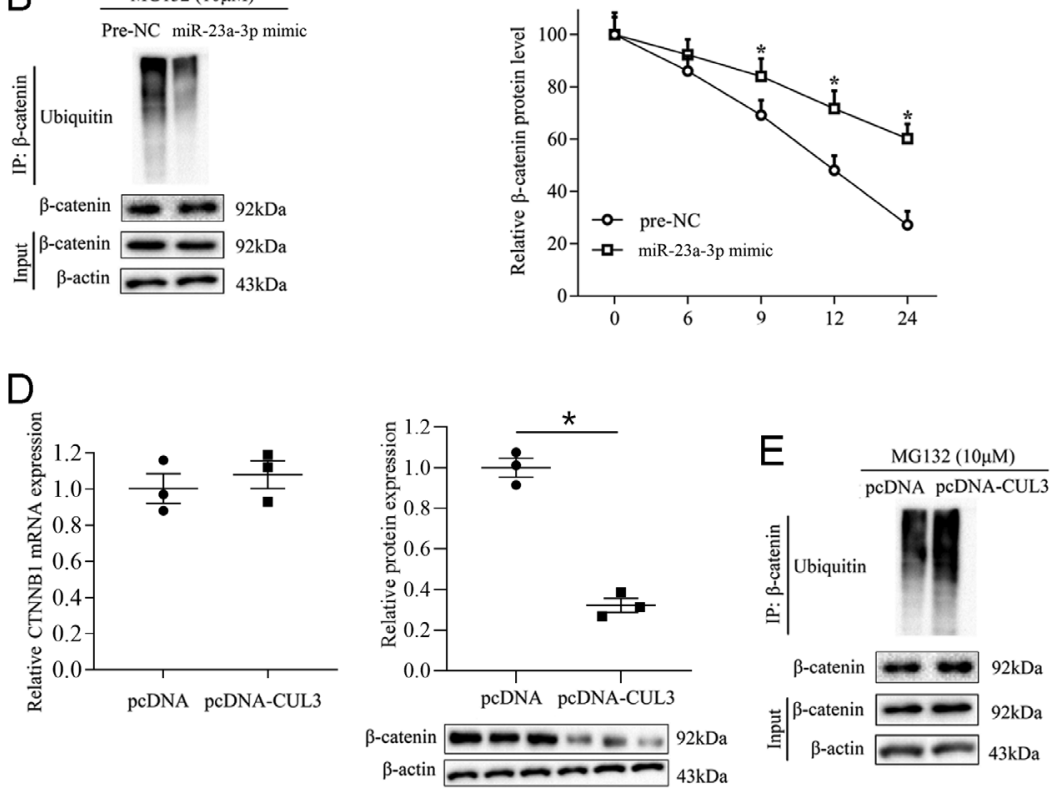
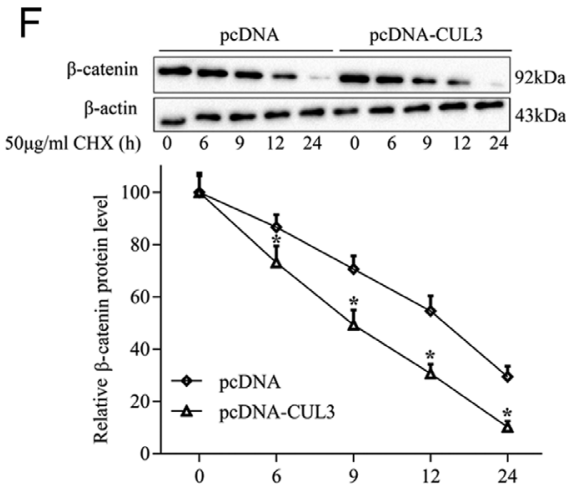

receptivity via CUL3/ $\beta$-catenin pathway, providing evidence for the mechanisms underlying endometrial receptivity and embryo implantation.

miRNAs are a subset of ncRNAs with a length of less than 20 nucleotides. The regulatory functions of miRNAs are mainly exerted by two ways (Beermann et al. 2016). On the one hand, by completely binding with the $3^{\prime} \mathrm{UTR}$ of targeted mRNA, miRNAs promote the degradation of the mRNA, thus inhibiting gene expressions. On the other hand, by partly binding with the 3'UTR of targeted mRNA, miRNAs inhibit translation of the mRNA, thus suppressing gene expressions. In our study, the regulatory function of miR-23a-3p on CUL3 was elucidated by using luciferase reporter assay, as the mutation in the

\section{Figure 4}

miR-23a-3p suppresses the ubiquitination of $\beta$-catenin via inhibiting CUL3 expression. (A) The IP assay was used to identify the binding between $\beta$-catenin and CUL3. (B) Ishikawa cells were transfected with miR-23a-3p mimic and $\mathrm{HA}-\mathrm{Ub}$ (Ubiquitin) with MG132 $(10 \mu \mathrm{g} / \mathrm{mL})$ treatment for $2 \mathrm{~h}$ before detection. The cell lysates were immunoprecipitated with anti- $\beta$-catenin, and the ubiquitination levels of $\beta$-catenin were detected by immunoblotting with anti-HA antibody. (C) Ishikawa cells were transfected with miR-23a-3p mimic and treated with $\mathrm{CHX}$ which is a protein synthesis inhibitor. The protein level of $\beta$-catenin was detected using Western blot analysis at 0, 6, 9, 12, and $24 \mathrm{~h}$ after CHX treatment. (D) Ishikawa cells were transfected with pCDNA-CUL3 to overexpress CUL3. The mRNA expression of CTNNB1 (the encoding gene of $\beta$-catenin) and the protein level of $\beta$-catenin were detected using qRT-PCR and Western blot analysis, respectively. (E) Ishikawa cells were transfected with pcDNA-CUL3 and HA-Ub with MG132 $(10 \mu \mathrm{g} / \mathrm{mL})$ treatment for $2 \mathrm{~h}$ before detection. The cell lysates were immunoprecipitated with anti- $\beta$ catenin, and the ubiquitination levels of $\beta$-catenin were detected by immunoblotting with anti-HA antibody. (F) Ishikawa cells were transfected with pCDNA-CUL3 and treated with CHX. The protein level of $\beta$-catenin was detected using Western blot analysis at $0,6,9,12$, and $24 \mathrm{~h}$ after $\mathrm{CHX}$ treatment. (G) Ishikawa cells were transfected with HA-Ub and miR-23a-3p inhibitor or co-transfected with siRNA-CUL3, followed by MG132 $(10 \mu \mathrm{g} / \mathrm{mL})$ treatment for $2 \mathrm{~h}$ before detection. The cell lysates were

immunoprecipitated with anti- $\beta$-catenin, and the ubiquitination levels of $\beta$-catenin were detected by immunoblotting with anti-HA antibody. $\star P<0.05$. predicted binding sites did not change the luciferase activities either overexpressing miR-23a-3p or knocking down miR-23a-3p, while the WT groups did change the luciferase activities. Additionally, studies have revealed that miRNAs can be sponged by long lncRNAs, which is known as the competing endogenous RNA (ceRNA) mechanism. miR-23a-3p was also reported to be sponged by some IncRNAs. For example, Zhao et al. (2019) demonstrated that IncRNA NEAT1 could compete with the mRNA to bind with miR-23a-3p, therefore negating the inhibition of miR-23a-3p to gene expressions. Since several lncRNAs, such as lncRNA ENST00000433673 (Li et al. 2019) and lncRNA PTENP1 (Takamura et al. 2019), have been reported to be involved in the regulation of 

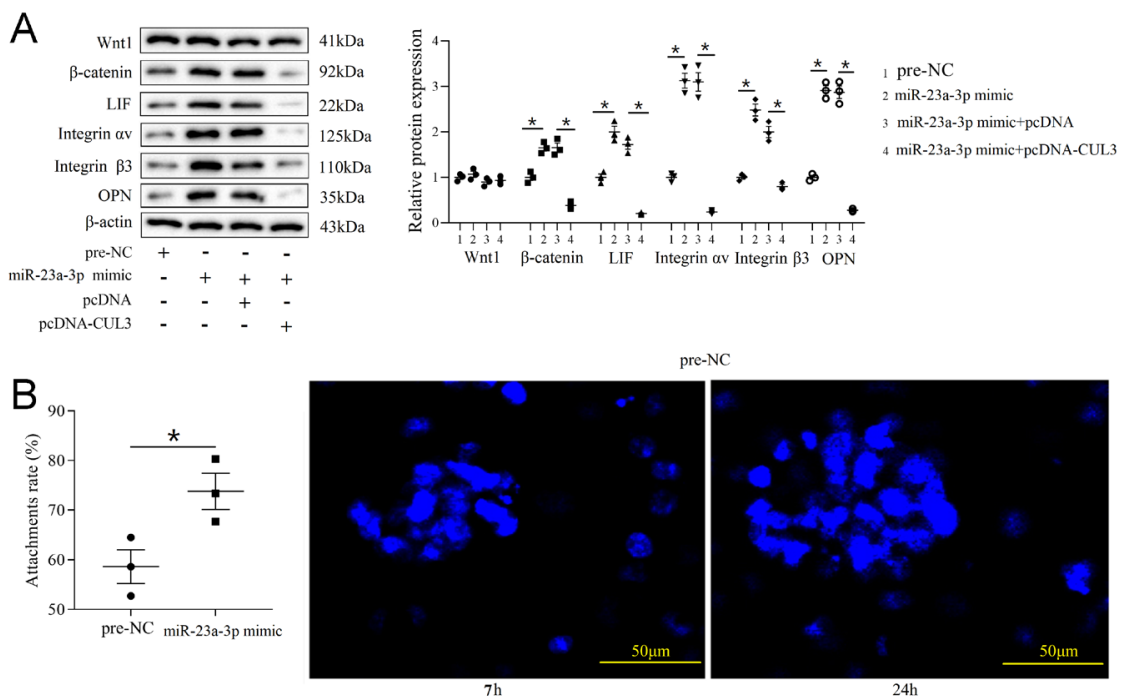

re-NC
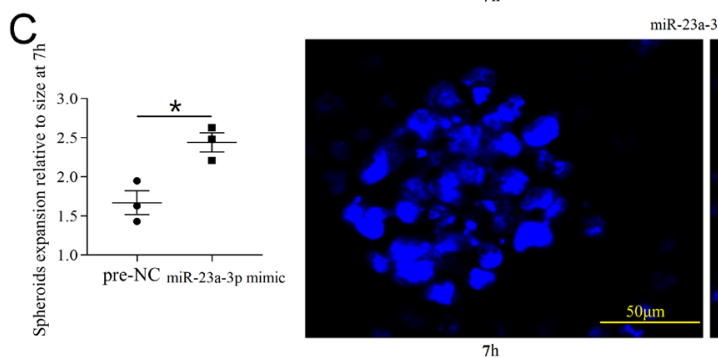

miR-23a-3p mimic

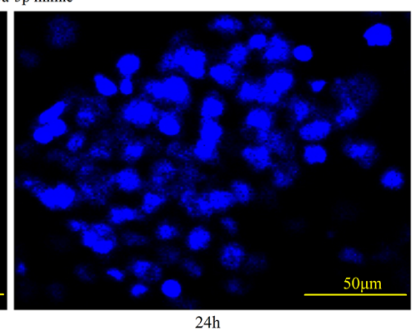

\section{Figure 5}

miR-23a-3p promotes JAR spheroid attachments in Ishikawa cells via suppressing CUL3 expression and increasing $\beta$-catenin expression. Ishikawa cells were transfected with miR-23a-3p mimic or co-transfected with CUL3 overexpressing plasmid (pcDNA-CUL3). (A) The levels of endometrial receptivity-proteins, including LIF, integrin $\alpha V$, integrin $\beta 3$, and OPN, and the protein levels of Wnt 1 and $\beta$-catenin were detected using Western blot analysis. (B) The spheroid attachments were performed by co-culturing human choriocarcinoma cell line (JAR, 45 spheroids/dish) with the Ishikawa cell monolayer after transfecting Ishikawa cells with miR-23a-3p mimic and the treatment of 10-8 $\mathrm{mol} / \mathrm{L} 17 \beta$-estradiol for $24 \mathrm{~h}$. The attachment rate was calculated as the ratio of the attached spheroid number to the seeded spheroid number. (C) Immunofluorescence analysis of JAR spheroids after the co-culture. The spheroid expansion was calculated as the ratio of the spheroid diameter at $24 \mathrm{~h}$ to the spheroid diameter at $7 \mathrm{~h} . * P<0.05$. embryo implantation. Whether the ceRNA mechanisms exist in the miR-23a-3p/CUL3 pathway deserves further investigations.

Ubiquitination involves a cascade of reactions which are catalyzed by ubiquitin enzymes, including E1, E2, and E3 (Zheng \& Shabek 2017). The ubiquitinactivating enzyme E1 transfers ubiquitin to the active site cysteine of the ubiquitin-conjugating enzyme E2. Then the ubiquitin ligase E3 transfers ubiquitin to the substrate from E2. Among them, Cullin-RING ligases (CRLs) are the most common family of E3 (Petroski \& Deshaies 2005). CUL3 is a subunit of CRLs that helps form a central stalk-like scaffold to constrain the substrate binding (Petroski \& Deshaies 2005). A previous study has revealed that CUL3 was lowly expressed in the placental villi from pre-eclamptic patients and that, in the maternal decidua, lack of CUL3 resulted in repressed invasion of trophoblast cells (Zhang et al. 2015). Their study suggested that CUL3 may play a critical role in the embryo implantation process. Since the ubiquitination of $\beta$-catenin is causally related to the poor endometrial receptivity and implantation failure (Hess et al. 2007, Liu et al. 2010), we supposed that the ubiquitin-associated protein CUL3 might affect embryo implantation process via modulating $\beta$-catenin. The overexpression of CUL3 increased the ubiquitin-bound $\beta$-catenin and the degradation speed, while the overexpression of miR-23a-3p reduced the ubiquitin-bound $\beta$-catenin and the degradation speed. Meanwhile, the simultaneous knockdown of miR-23a-3p and CUL3 negated the promotion of $\beta$-catenin ubiquitination caused by miR-23a-3p knockdown. Our findings demonstrated that the $\beta$-catenin ubiquitination was modulated by the miR-23a-3p/CUL3 pathway. Notably, the expression of Wnt1 was not affected by the miR-23a-3p/CUL3 pathway in the current study. However, Wnt proteins have been reported to participate in blastocyst development and implantation both in mice and human (Taylor 2000, Mohamed et al. 2004). More evidence is still needed on the effects of Wnt protein in implantation regulation.

Inevitably, several limitations exist in our study. The co-culture model is just a surrogate which may not fully represent embryo implantation in vivo, and a lack of in vivo animal studies is a main limitation of this study. In the current study, the experiments with human EECs and blastocysts have shown that overexpression of miR-23a-3p promotes JAR spheroid attachments in Ishikawa cells, strongly proving the benefit of miR-23a-3p for the endometrial receptivity and embryo implantation. In consideration of the high conservation of miR-23a-3p 
and its CUL3-binding site in human and murine, we did not repeat the experiments in murine. Experiments with murine EECs and blastocysts maybe a good supplementary, before an available animal model developed, which requires further efforts.

In conclusion, our study indicated that miR-23a-3p is beneficial for improving endometrial receptivity and trophoblast attachments during embryo implantation. Meanwhile, miR-23a-3p suppressed ubiquitination of $\beta$-catenin via inhibiting CUL3 expression. Our findings provide a novel insight into the mechanisms underlying embryo implantation.

\section{Declaration of interest}

The authors declare that there is no conflict of interest that could be perceived as prejudicing the impartiality of the research reported.

\section{Funding}

This study was supported by the National Natural Science Foundation of China (Grant No. 81701448).

\section{Ethics approval and consent to participate}

This study was approved by the Institute Research Medical Ethics Committee of The First Affiliated Hospital of Zhengzhou University.

\section{Availability of data and material}

The datasets used and/or analyzed during the current study are available from the corresponding author on reasonable request.

\section{References}

Angers S, Thorpe CJ, Biechele TL, Goldenberg SJ, Zheng N, MacCoss MJ \& Moon RT 2006 The KLHL12-Cullin-3 ubiquitin ligase negatively regulates the Wnt-beta-catenin pathway by targeting dishevelled for degradation. Nature Cell Biology 8 348-357. (https://doi.org/10.1038/ ncb1381)

Beermann J, Piccoli MT, Viereck J \& Thum T 2016 Non-coding RNAs in development and disease: background, mechanisms, and therapeutic approaches. Physiological Reviews 96 1297-1325. (https://doi. org/10.1152/physrev.00041.2015)

Berneau SC, Ruane PT, Brison DR, Kimber SJ, Westwood M \& Aplin JD 2019 Characterisation of osteopontin in an in vitro model of embryo implantation. Cells 8 432. (https://doi.org/10.3390/ cells8050432)

Cagil N, Ugurlu N, Cakmak HB, Ilker Kocamis S, Turak D \& Simsek S 2011 Photorefractive keratectomy in treatment of refractive amblyopia in the adult population. Journal of Cataract and Refractive Surgery 37 2167-2174. (https://doi.org/10.1016/j.jcrs.2011.06.026)

Chakrabarty A, Tranguch S, Daikoku T, Jensen K, Furneaux H \& Dey SK 2007 MicroRNA regulation of cyclooxygenase-2 during embryo implantation. PNAS 104 15144-15149. (https://doi.org/10.1073/ pnas.0705917104)
Diedrich K, Fauser BC, Devroey P, Griesinger G \& Evian Annual Reproduction (EVAR) Workshop Group 2007 The role of the endometrium and embryo in human implantation. Human Reproduction Update 13 365-377. (https://doi.org/10.1093/humupd/ dmm011)

Garrido-Gomez T, Dominguez F, Quinonero A, Estella C, Vilella F, Pellicer A \& Simon C 2012 Annexin A2 is critical for embryo adhesiveness to the human endometrium by RhoA activation through F-actin regulation. FASEB Journal 26 3715-3727. (https://doi. org/10.1096/fj.12-204008)

Godfrey TC, Wildman BJ, Beloti MM, Kemper AG, Ferraz EP, Roy B, Rehan M, Afreen LH, Kim E, Lengner CJ, et al. 2018 The microRNA23a cluster regulates the developmental HoxA cluster function during osteoblast differentiation. Journal of Biological Chemistry 293 17646-17660. (https://doi.org/10.1074/jbc.RA118.003052)

Haouzi D, Mahmoud K, Fourar M, Bendhaou K, Dechaud H, De Vos J, Reme T, Dewailly D \& Hamamah S 2009 Identification of new biomarkers of human endometrial receptivity in the natural cycle. Human Reproduction 24 198-205. (https://doi.org/10.1093/humrep/ den360)

Hess AP, Hamilton AE, Talbi S, Dosiou C, Nyegaard M, Nayak N, Genbecev-Krtolica O, Mavrogianis P, Ferrer K, Kruessel J, et al. 2007 Decidual stromal cell response to paracrine signals from the trophoblast: amplification of immune and angiogenic modulators Biology of Reproduction 76 102-117. (https://doi.org/10.1095/ biolreprod.106.054791)

Hu SJ, Ren G, Liu JL, Zhao ZA, Yu YS, Su RW, Ma XH, Ni H, Lei W \& Yang ZM 2008 MicroRNA expression and regulation in mouse uterus during embryo implantation. Journal of Biological Chemistry $\mathbf{2 8 3}$ 23473-23484. (https://doi.org/10.1074/jbc.M800406200)

Li D, Jiang W, Jiang Y, Wang S, Fang J, Zhu L, Zhu Y, Yan G, Sun H, Chen L, et al. 2019 Preliminary functional inquiry of lncRNA ENST00000433673 in embryo implantation using bioinformatics analysis. Systems Biology in Reproductive Medicine 65 164-173. (https:// doi.org/10.1080/19396368.2018.1563844)

Li HWR, Li YX, Li TT, Fan H, Ng EH, Yeung WS, Ho PC \& Lee KF 2017 Effect of ulipristal acetate and mifepristone at emergency contraception dose on the embryo-endometrial attachment using an in vitro human trophoblastic spheroid and endometrial cell co-culture model. Human Reproduction 32 2414-2422. (https://doi. org/10.1093/humrep/dex328)

Lim HJ \& Wang H 2010 Uterine disorders and pregnancy complications: insights from mouse models. Journal of Clinical Investigation 120 1004-1015. (https://doi.org/10.1172/JCI41210)

Liu C, Li Y, Semenov M, Han C, Baeg GH, Tan Y, Zhang Z, Lin X \& He X 2002 Control of beta-catenin phosphorylation/degradation by a dual-kinase mechanism. Cell 108 837-847. (https://doi. org/10.1016/s0092-8674(02)00685-2)

Liu Y, Kodithuwakku SP, Ng PY, Chai J, Ng EH, Yeung WS, Ho PC \& Lee KF 2010 Excessive ovarian stimulation up-regulates the Wntsignaling molecule DKK1 in human endometrium and may affect implantation: an in vitro co-culture study. Human Reproduction $\mathbf{2 5}$ 479-490. (https://doi.org/10.1093/humrep/dep429)

Mohamed OA, Dufort D \& Clarke HJ 2004 Expression and estradiol regulation of Wnt genes in the mouse blastocyst identify a candidate pathway for embryo-maternal signaling at implantation. Biology of Reproduction 71 417-424. (https://doi.org/10.1095/ biolreprod.103.025692)

Pan JL, Yuan DZ, Zhao YB, Nie L, Lei Y, Liu M, Long Y, Zhang JH, Blok LJ, Burger CW, et al. 2017 Progesterone-induced miR-133a inhibits the proliferation of endometrial epithelial cells. Acta Physiologica 219 683-692. (https://doi.org/10.1111/apha.12762)

Petroski MD \& Deshaies RJ 2005 Function and regulation of cullin-RING ubiquitin ligases. Nature Reviews: Molecular Cell Biology 6 9-20. (https://doi.org/10.1038/nrm1547) (c) 2020 Society for Endocrinology Published by Bioscientifica Ltd. Printed in Great Britain 
Schmitz C, Yu L, Bocca S, Anderson S, Cunha-Filho JS, Rhavi BS \& Oehninger S 2014 Role for the endometrial epithelial protein MFGE8 and its receptor integrin alphavbeta3 in human implantation: results of an in vitro trophoblast attachment study using established human cell lines. Fertility and Sterility 101 874-882. (https://doi. org/10.1016/j.fertnstert.2013.12.015)

Sonderegger S, Pollheimer J \& Knofler M 2010 Wnt signalling in implantation, decidualisation and placental differentiation - review. Placenta 31 839-847. (https://doi.org/10.1016/j.placenta.2010.07.011)

Takamura M, Zhou W, Rombauts L \& Dimitriadis E 2019 The long noncoding RNA PTENP1 regulates human endometrial epithelial adhesive capacity in vitro: implications in infertility. Biology of Reproduction 102 53-62. (https://doi.org/10.1093/biolre/ioz173)

Taylor HS 2000 The role of HOX genes in human implantation. Human Reproduction Update 6 75-79. (https://doi.org/10.1093/ humupd/6.1.75)

Wang J, Ke L, Jiang X-1 \& Zhang C 2018 A review of microRNAs related to the occurrence, diagnosis, and prognosis of non-small cell lung cancer. Clinical Surgery Research Communications 2 1-8. (https://doi. org/10.31491/CSRC.2018.9.017)

Yang Q, Gu WW, Gu Y, Yan NN, Mao YY, Zhen XX, Wang JM, Yang J, Shi HJ, Zhang X, et al. 2018 Association of the peripheral blood levels of circulating microRNAs with both recurrent miscarriage and the outcomes of embryo transfer in an in vitro fertilization process.
Journal of Translational Medicine 16 186. (https://doi.org/10.1186/ s12967-018-1556-x)

Zhang Q, Yu S, Huang X, Tan Y, Zhu C, Wang YL, Wang H, Lin HY, Fu J \& Wang H 2015 New insights into the function of cullin 3 in trophoblast invasion and migration. Reproduction 150 139-149. (https://doi.org/10.1530/REP-15-0126)

Zhao C, Wang S, Zhao Y, Du F, Wang W, Lv P \& Qi L 2019 Long noncoding RNA NEAT1 modulates cell proliferation and apoptosis by regulating miR-23a-3p/SMC1A in acute myeloid leukemia. Journal of Cellular Physiology 234 6161-6172. (https://doi.org/10.1002/ jcp.27393)

Zheng N \& Shabek N 2017 Ubiquitin ligases: structure, function, and regulation. Annual Review of Biochemistry 86 129-157. (https://doi. org/10.1146/annurev-biochem-060815-014922)

Zheng Q, Dai K, Cui X, Yu M, Yang X, Yan B, Liu S \& Yan Q 2016 Leukemia inhibitory factor promote trophoblast invasion via urokinase-type plasminogen activator receptor in preeclampsia. Biomedicine and Pharmacotherapy 80 102-108. (https://doi. org/10.1016/j.biopha.2016.03.005)

Zhu LH, Sun LH, Hu YL, Jiang Y, Liu HY, Shen XY, Jin XY, Zhen X, Sun HX \& Yan GJ 2013 PCAF impairs endometrial receptivity and embryo implantation by down-regulating beta3-integrin expression via HOXA10 acetylation. Journal of Clinical Endocrinology and Metabolism 98 4417-4428. (https://doi.org/10.1210/jc.2013-1429)

Received in final form 9 June 2020

Accepted 26 June 2020

Accepted Manuscript published online 26 June 2020
(C) 2020 Society for Endocrinology Published by Bioscientifica Ltd. Printed in Great Britain 